\title{
A Continuous-Time Delta-Sigma ADC for Portable Ultrasound Scanners
}

\author{
Llimos Muntal, Pere; Jørgensen, Ivan Harald Holger; Bruun, Erik
}

Published in:

Analog Integrated Circuits and Signal Processing

Link to article, DOI:

10.1007/s10470-017-1004-5

Publication date:

2017

Document Version

Peer reviewed version

Link back to DTU Orbit

Citation $(A P A)$ :

Llimos Muntal, P., Jørgensen, I. H. H., \& Bruun, E. (2017). A Continuous-Time Delta-Sigma ADC for Portable Ultrasound Scanners. Analog Integrated Circuits and Signal Processing, 92(3), 393-402.

https://doi.org/10.1007/s10470-017-1004-5

\section{General rights}

Copyright and moral rights for the publications made accessible in the public portal are retained by the authors and/or other copyright owners and it is a condition of accessing publications that users recognise and abide by the legal requirements associated with these rights.

- Users may download and print one copy of any publication from the public portal for the purpose of private study or research.

- You may not further distribute the material or use it for any profit-making activity or commercial gain

- You may freely distribute the URL identifying the publication in the public portal

If you believe that this document breaches copyright please contact us providing details, and we will remove access to the work immediately and investigate your claim. 


\title{
A Continuous-Time Delta-Sigma ADC for Portable Ultrasound Scanners
}

\author{
Pere Llimós Muntal • Ivan H.H. \\ Jørgensen • Erik Bruun
}

Received: date / Accepted: date

\begin{abstract}
A fully differential fourth-order 1-bit continuous-time delta-sigma ADC designed in a $65 \mathrm{~nm}$ process for portable ultrasound scanners is presented in this paper. The circuit design, implementation and measurements on the fabricated die are shown. The loop filter consists of RC-integrators, programmable capacitor arrays, resistors and voltage feedback DACs. The quantizer contains a pulse generator, a high-speed clocked comparator and a pull-down clocked latch to ensure constant delay in the feedback loop. Using this implementation, a small and low-power solution required for portable ultrasound scanner applications is achieved. The converter has a supply voltage of $1.2 \mathrm{~V}$, a bandwidth of $10 \mathrm{MHz}$ and an oversampling ratio of 16 leading to an operating frequency of $320 \mathrm{MHz}$. The design occupies a die area of $0.0175 \mathrm{~mm}^{2}$. Simulations with extracted parasitics show a SNR of $45.2 \mathrm{~dB}$ and a current consumption of $489 \mu \mathrm{A}$. However, by adding a model of the measurement setup used, the performance degrades to $42.1 \mathrm{~dB}$. The measured SNR and current consumption are $41.6 \mathrm{~dB}$ and $495 \mu \mathrm{A}$, which closely fit with the expected sim-
\end{abstract}

Pere Llimós Muntal

Elektrovej, DTU - Building 325

2800 Kongens Lyngby

Denmark

Tel.: +45 45252525

E-mail: plmu@elektro.dtu.dk

Ivan H.H. Jørgensen

Elektrovej, DTU - Building 325

2800 Kongens Lyngby

Denmark

E-mail: ihhj@elektro.dtu.dk

Erik Bruun

Elektrovej, DTU - Building 325

2800 Kongens Lyngby

Denmark

E-mail: eb@elektro.dtu.dk 
ulations. Several dies have been measured, and an estimation of the die spread distribution is given.

Keywords Ultrasound · Portable · Scanners · Integrated · Continuous-Time • Delta-Sigma · ADC

\section{Introduction}

Ultrasound scanning is a widely used technique in medical applications due to its operating simplicity, non-invasive nature, live imaging capabilities and extended diagnosis range. However, the commonly used static ultrasound scanners are expensive, large and have no power consumption limitations since they are plugged into the AC mains. Due to its virtually unlimited supply power, the electronics of a static scanner are generic discrete components which typically have a higher performance than required and thus use excessive power.

In the last decade, portable ultrasound scanners have been emerging. Research on their implementation has increased since they are a small and flexible alternative to traditional static ultrasound scanners. There are several challenges in the design of a portable ultrasound scanner. Firstly, due to the reduced size, the maximum power dissipation on the scanner is approximately $2 \mathrm{~W}$ to guarantee that it does not become so heated that it is uncomfortable to handle. Secondly, since the device is battery supplied, the maximum power consumption of the electronics is also limited, which obsoletes the usage of generic discrete components. An application specific integrated circuit (ASIC) solution is required to custom design the electronics and minimize the are and power consumption. Implementing the electronics using ASICs leads to the best signal-to-noise ratio (SNR) for the available area and power budget, which directly translates into the best picture quality achievable.

A portable ultrasound scanner comprises several channels, and each of them consist of a transducer, a transmitting circuit $(\mathrm{Tx})$ and a receiving circuit $(\mathrm{Rx})$. The Tx excites the transducer with high-voltage signals in order to generate ultrasound waves. The Rx amplifies, delays and digitizes the signal induced in the transducer by the reflected waves. Since both size and power are critical to achieve a handheld device, all blocks need to be optimized accordingly.

In this paper, the design, implementation and measurements of a fully differential fourth-order 1-bit continuous-time delta-sigma analog-to-digital converter (CTDS ADC) are presented. A continuous-time implementation was chosen over a discrete-time one due to the required high operating frequency and low power requirements $[1,2]$. Optimized performance is needed to achieve a usable picture quality, which was studied in [3]. The circuit is designed to be used in a 64-channel synthetic aperture sequential beamforming (SASB) [4-7] portable ultrasound scanner based on capacitive micromachined ultrasonic transducers (CMUTs). The circuit is fabricated in a $65 \mathrm{~nm}$ process with a supply voltage of $1.2 \mathrm{~V}$. 


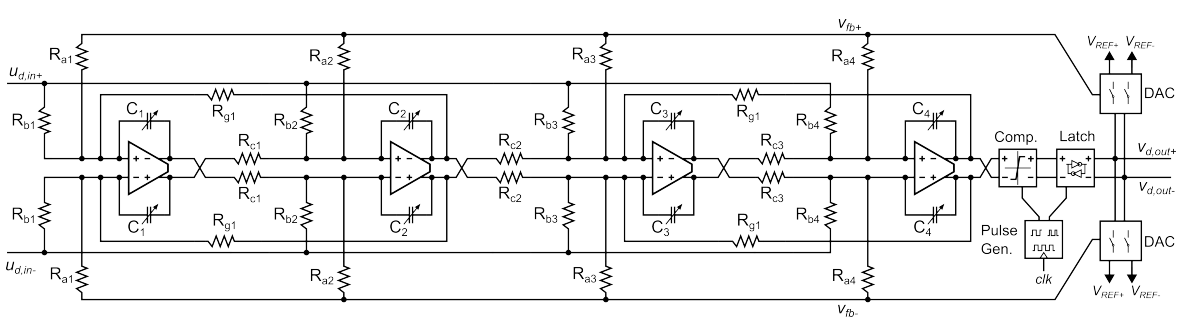

Fig. 1 Structure of the fully differential fourth-order 1-bit continuous-time delta-sigma $\mathrm{ADC}$ with two resonators for optimal zero placing.

\section{CTDS ADC topology and specifications}

In [8], the 64-channel portable ultrasound scanner system based on CMUTs was studied and the most adequate topology and specifications were derived. A fourth-order 1-bit CTDS ADC topology was chosen and a summary of the SNR, bandwidth (BW), oversampling ratio (OSR), supply voltages $\left(V_{S S} / V_{D D}\right)$, common mode level $\left(V_{C M}\right)$ and maximum differential input voltage $\left(u_{i n, \max }\right)$ is shown in Table 1. The relatively low SNR requirement for the ADC is possible due to the in-handle SASB [4-7] of the scanner, which sums the 64 channels into one. This technique requires a fine delay accuracy of $3.125 \mathrm{~ns}$ in order to temporally align the signals from each channel before summing them. As a consequence, a CTDS ADC implementation was chosen over a Nyquist rate ADC. Using a $10 \mathrm{MHz}$ bandwidth Nyquist rate ADC would require interpolation filters to achieve the required delay accuracy. These type of filters are complex, process dependent and highly area and power demanding, which is not acceptable for portable ultrasound scanner applications. Contrarily, a 1-bit oversampled converter at a sampling frequency of $320 \mathrm{MHz}$ inherently embeds the necessary delay accuracy in the bit stream. As a result, a simple and efficient inverter-based clocked digital delay line with switches can be used as a delay block.

Due to the low SNR requirements, the thermal noise was found to be negligible compared to the inherent quantization noise, which is rare in CTDS ADC design. Typically, the signal to quantization noise ratio (SQNR) of the CTDS ADC is designed 10-12dB higher than the specified SNR to accommodate for thermal noise, non-idealities and transistor level implementation limitations. In this design, the thermal noise can be neglected which, as it is can be seen later, affects significantly the design choices and implementation of the CTDS ADC. Due to the low impact of the thermal noise, all the tradeoffs of the design have been biased towards low current consumption instead of noise performance. Furthermore, it allows for more headroom to be used to accommodate non-idealities and transistor level implementation limitations. Note that the main goal is to find the design with the lowest current consumption that satisfies the specifications. Therefore, the SNR will be purposely degraded by reducing the current until the targeted SNR is reached. 
Table 1 Continuous-Time Delta-Sigma ADC Specifications

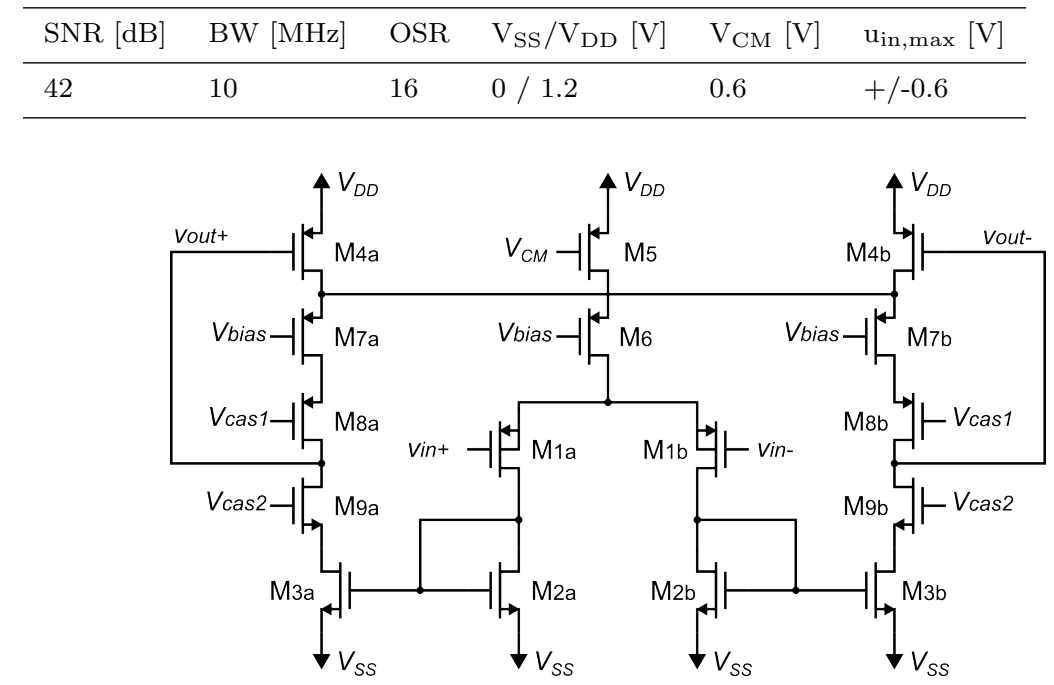

Fig. 2 Symmetrical OTA schematic with cascodes and CMFB.

The block level structure of the fully differential CTDS ADC designed is shown in Fig. 1, where the input and output are $u_{i n}=u_{d, i n+}-u_{d, \text { in }}$ and $v_{\text {out }}=v_{d, \text { out }+}-v_{d, \text { out }}$ respectively. The loop filter consists of four operational transconductance amplifier (OTA) based RC-integrators, resistor coefficients and two feedback voltage digital-to-analog converters (DACs). The $\mathrm{RC}$-integrators are grouped in pairs in order to create two resonators, which optimally place the zeros of the transfer function to improve the SQNR. The design was done according to the optimization method shown in [9]. Additionally, the integrating capacitors are designed to be programmable to be able to compensate for corners and process variations. The quantizer is implemented with a pulse generator, a high-speed clocked comparator and a pull-down clocked latch. In the next section, the design of each of the blocks of the CTDS ADC is thoroughly described.

\section{Block design}

In this section, the design of each block of the CTDS ADC is shown. In each subsection, the specifications, topology and design choices of the block are discussed. Circuitry for portable ultrasound scanners has to be small and efficient, therefore, all the blocks of the ADC are designed to minimize its die area and power consumption. Note that in all schematics, the bulks of the PMOS and NMOS transistors are connected to the positive supply $\left(V_{D D}\right)$ and negative supply $\left(V_{S S}\right)$ respectively if it is not indicated otherwise. 


\subsection{Operational Transconductance Amplifier}

The specifications for the OTA are a gain $\left(\mathrm{A}_{\mathrm{V}}\right)$ of $40 \mathrm{~dB}$, a gain-bandwidth $(\mathrm{GBW})$ of $1.3 \mathrm{GHz}$, phase margin (PM) of $35^{\circ}$ and a slew rate $(\mathrm{SR})$ of $120 \mathrm{~V} / \mathrm{us}$. The load of the OTA is the sum of the nominal value of the integrating capacitor and the parasitics of the circuitry. The value of the nominal capacitance was chosen to be small in order to minimize the current consumption of the OTA. As a result, the coefficient resistors are large and noisy. Typically, this is a problem in CTDS ADCs since the thermal noise limits the SNR. Much smaller resistor coefficients need to be used, increasing the size of the capacitors and thereby and the current in the OTAs. Nonetheless, the SNR of this design is not thermal noise limited, hence, the integrating capacitors should be chosen as small as possible to achieve a power efficient design. The nominal value of the integrating capacitors was chosen to be $100 \mathrm{fF}$ leading to resistor coefficients in the order of $100 \mathrm{k} \Omega$. Smaller capacitor values were not used to not compromise the matching and parasitic sensitivity of the design.

The most demanding parameter of the specifications is the GBW, and it needs to be achieved with the minimum current possible. The symmetrical OTA topology shown in Fig. 2 is selected since it has a very high currentto-GBW ratio, low offset, high output swing, is symmetrical and tolerant to mismatch [10]. Cascoded transistors $M_{8 a} / M_{8 b}$ and $M_{9 a} / M_{9 b}$ have to be added to boost the gain. The cascodes limit the output swing of the OTA, however, this is not an issue since the output swing of the OTA-based integrators can be adjusted by scaling the filter coefficients. The main disadvantage of symmetrical OTAs is the high levels of thermal noise, however, as it was stated before, due to the low SNR required, the thermal noise is not a limiting factor. The bias current in the inner branch is generated by $M_{6}$ and is mirrored five times larger with the current mirror formed by $M_{2 a} / M_{2 b}$ and $M_{3 a} / M_{3 b}$. The common-mode feedback (CMFB) consists of $M_{4 a} / M_{4 b}$ and $M_{5}$, which detects the output levels and adjusts the current in the outer branches to compensate for it.

The OTA was simulated with extracted parasitics including process variations and mismatch and the performance obtained is shown in Table 2. The nominal value and the maximum and minimum value simulated across all the process variations and mismatch are noted. All the specifications are satisfied even in the worst case of each parameter. A small margin for each parameter is left for any post-silicon variations. Note that the same OTA design is used for all four integrators. However, in future versions of the CTDS ADC the current in the second, third and fourth OTAs will be scaled down due to their lower requirements.

\subsection{Programmable capacitor array}

Due to process variations, the value of the resistors and capacitors can range up to $+/-20 \%$ in the worst case corners. Therefore, the coefficients of the 
Table 2 Symmetrical OTA Performance over Corners and Variations

\begin{tabular}{lllll}
\hline & $\mathrm{A}_{\mathrm{v}}[\mathrm{dB}]$ & $\mathrm{GBW}[\mathrm{GHz}]$ & $\mathrm{PM}\left[^{\circ}\right]$ & $\mathrm{SR}[\mathrm{V} / \mathrm{\mu s}]$ \\
\hline Nom. & 46.3 & 1.41 & 40.6 & 267 \\
Min. & 45.9 & 1.35 & 39.5 & 256 \\
Max. & 46.6 & 1.44 & 41.6 & 277 \\
\hline
\end{tabular}
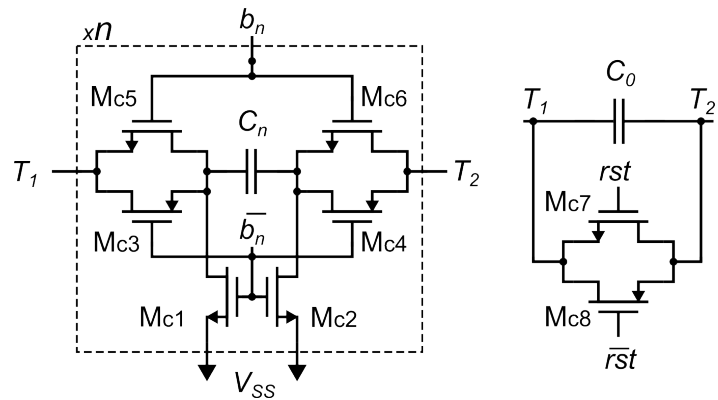

Fig. 3 Programmable capacitor array schematic. Nominal capacitance of $100 \mathrm{fF}$ and $\mathrm{n}=3$.

loop filter, which depend inversely on the RC product, can vary significantly resulting in performance degradation and even instability. In order to compensate for these variations, the $100 \mathrm{fF}$ integrating capacitors are implemented as programmable capacitor array so that the capacitance value can be adjusted. The schematic of the array can be seen in Fig. 3 . The bits $b_{n}$ control whether the corresponding capacitor $C_{n}$ is connected to the input/output of the OTA or if it is disconnected and shorted to ground. In this design three control bits $(\mathrm{n}=1,2,3)$ are used, leading to eight possible capacitor values combining $C_{0}=60 \mathrm{fF}, C_{1}=10 \mathrm{fF}, C_{2}=20 \mathrm{fF}$ and $C_{3}=40 \mathrm{fF}$. The extra control bit, $r s t$, works as a reset signal of the CTDS ADC by shorting the input/output of the OTAs.

\subsection{High-speed clocked comparator}

The sampling frequency of the oversampled ADC is $320 \mathrm{MHz}$, therefore, a very fast comparator is needed. Furthermore, in order to get consistent comparisons with the same starting state, the comparator needs to be reset every cycle. The topology used is the one suggested in [11], and it is shown in Fig. 4. Two small inverters acting as buffers are added at the comparator outputs, $v_{o+}$ and $v_{o-}$, to load them equally. As a result, the comparator behaves more equally for both comparator decisions. The comparator has two different phases. Firstly, when the clock $c l k_{c}$ is low, the comparator is disabled and both outputs $v_{o+}$ and $v_{o-}$ are pulled up to $V_{D D}$. Secondly, when $c l k_{c}$ goes high, the starting state of the comparator is unstable since both $v_{o+}$ and $v_{o-}$ are high. A small differential signal in the input pair of the comparator, $M_{10 a} / M_{10 b}$ will pull down either $v_{o+}$ or $v_{o-}$ through the two positive feedback paths formed by 


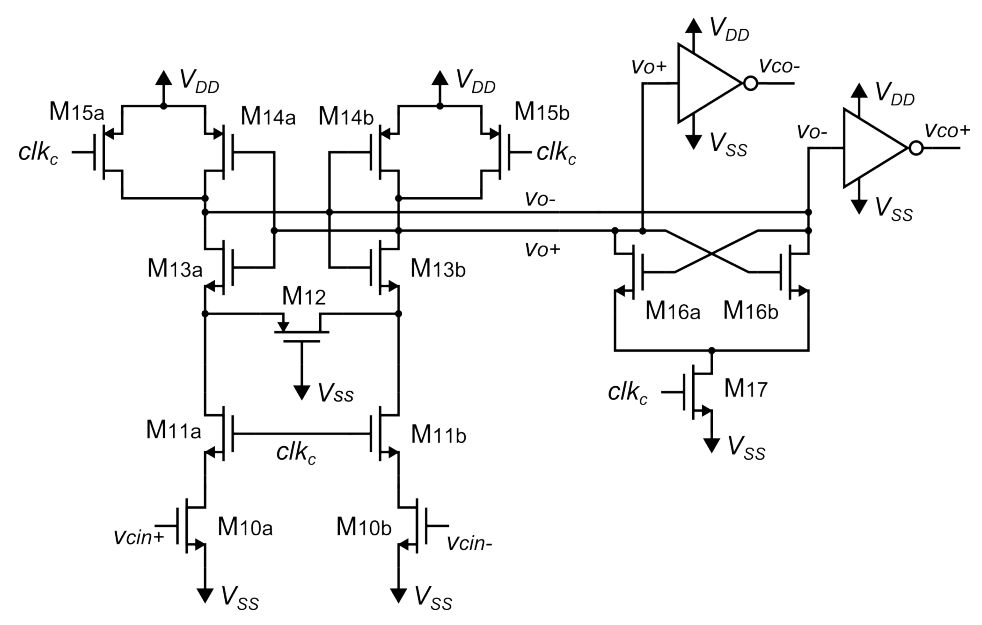

Fig. 4 Comparator schematic.

$M_{13 a} / M_{13 b}$ and $M_{16 a} / M_{16 b} . M_{14 a} / M_{14 b}$ are sized significantly bigger than the rest of the transistors so that once the circuit is flipped to one side, the input signal can not change the state allowing only one comparison per reset cycle.

\subsection{Pull-down clocked latch}

Even though the comparator is symmetric and equally loaded, the amplitude of its differential input signal determines the comparison time. The comparator takes longer to compare small differential signals, and is quicker at deciding for larger differential signals. This would result in varying delays in the feedback signals, which would decrease the SNR and could even cause instability. Hence, a clocked latch is needed. The latch provides a time consistent output independently of the comparator behaviour. Firstly, $c l k_{c}$ enables the comparator and after a decision time, $c l k_{l}$ enables the latch passing the comparator decision to the outputs of the CTDS ADC $v_{d, \text { out }}$ and $v_{d, \text { out }}$. The outputs are consistently generated on the rising edge of $c l k_{l}$, hence, the feedback signals are synchronized and have a constant delay.

The schematic of the pull-down clocked latch can be seen in Fig. 5. It consists of a latch formed by $M_{20 a} / M_{20 b}$ and $M_{21 a} / M_{21 b}$ and two pull down branches composed of $M_{18 a} / M_{18 b}$ and $M_{19 a} / M_{19 b}$. When the clock $c l k_{l}$ is low, both branches are disconnected, and the latch maintains its state. When $c l k_{l}$ is high, one of the branches pulls down one of the nodes of the latch forcing a state. The pulling strength of both branches is consistent every cycle since $v_{c o+}$ and $v_{c o-}$ are always either $V_{D D}$ or $V_{S S}$ when the latch is enabled. 


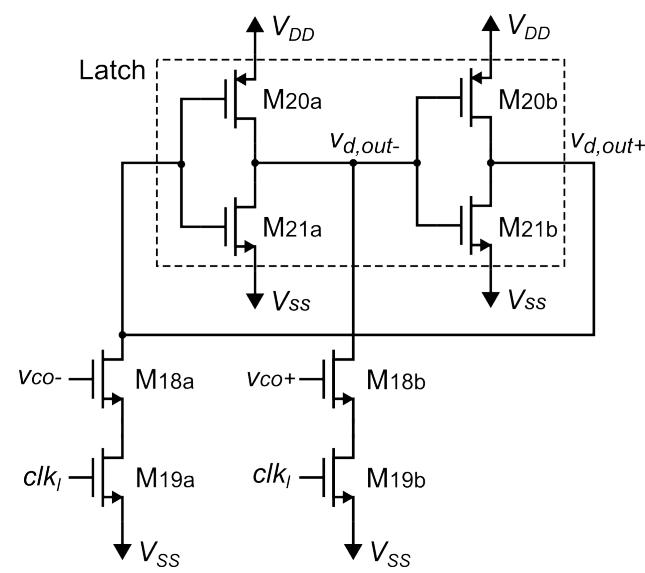

Fig. 5 Latch schematic.

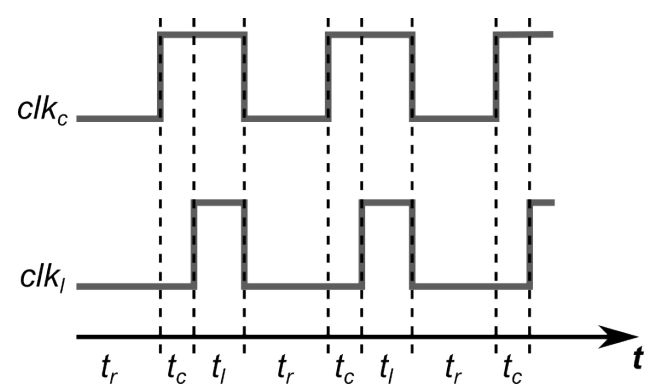

Fig. 6 Comparator and latch timing diagram.

\subsection{Pulse generator}

In order to control both the comparator and the latch the enabling pulses $c l k_{c}$ and $c l k_{l}$ need to be generated. As it is shown in Fig. 6, there are three states per cycle, the comparison time $\left(t_{c}\right)$, the latch time $\left(t_{l}\right)$ and the reset time $\left(t_{r}\right)$. During $t_{c}$, only the comparator is enabled. During $t_{l}$, both the comparator and latch are enabled. Finally, during $t_{r}$, both comparator and latch are disabled. It is important to notice that the comparator can stay enabled during the latch time since $M_{14 a} / M_{14 b}$ are designed to be very strong, hence the comparator inputs can not flip its outputs. This allows for a way simpler and more robust control scheme where it is not critical to turn off the comparator before the output is latched.

The pulse generator is implemented with a simple inverter delay line, and logic gates that generate $c l k_{c}$ and $c l k_{l}$ as shown in Fig. 7. Note that the input clock, $c l k_{i n}$, is buffered internally in order to minimize the routing effects from the clock source to the die. This simple design consumes a small current and is resistant to process and mismatch variations since, even though $t_{c}, t_{l}$ and $t_{r}$ can vary, these states can not overlap due to its inherent structure. 


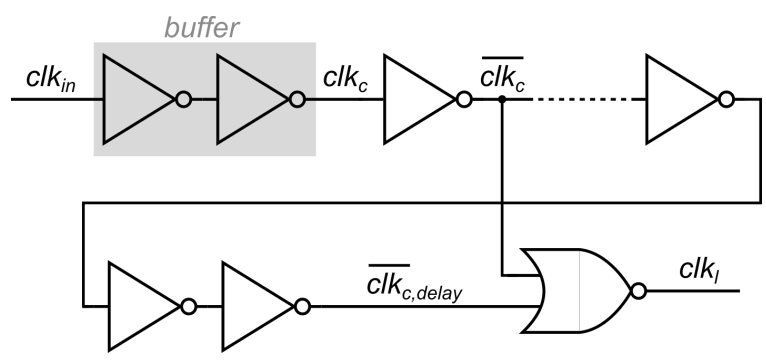

Fig. 7 Pulse generator schematic.
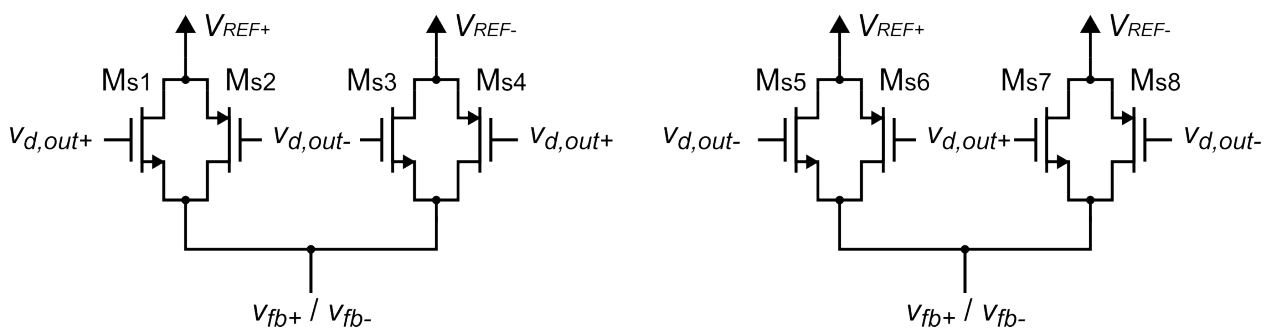

Fig. 8 Schematic of the voltage feedback DACs.

The loop delay of the CDTS ADC from the comparator to the DACs is largely dominated by $t_{c}$, which is determined by the delay across the inverter delay line. The layout of this block affects the unit delay of an inverter, therefore all the timing simulations need to be done with extracted parasitics. Following the specifications found through simulations in [8], the loop delay can not be higher than 300 ps. Simulations with extracted parastics of the implementation, including corners and mismatch variations, show that the loop delay varies from $210 \mathrm{ps}$ to $298 \mathrm{ps}$ with a nominal value of $252 \mathrm{ps}$, which satisfies the specifications.

\subsection{Voltage feedback DAC}

The two DACs of the system are chosen to be implemented as simple voltage DACs for simplicity, easiness of matching and area reduction. They consist of a PMOS and NMOS forming a transmission gate that connects the feedback nodes $v_{f b+} / v_{f b-}$ to either $V_{R E F+}=1.1 \mathrm{~V}$ ) or $V_{R E F-}=0.1 \mathrm{~V}$, depending on the gate signals $v_{d, \text { out }}$ and $v_{d, \text { out }}$ (Fig. 8). These transmission gates need to be fast, therefore small transistors should be used. Due to the large coefficient resistors in the order of $100 \mathrm{k} \Omega$, the on-resistance of the small transistors is neglectable. In order to obtain consistent, symmetric feedback pulses, both DACs should match, hence, several unit size transistors are used in each MOSFET device. The total width and length used for the DAC transistors are $W / L=200 / 60 \mathrm{~nm}$. 


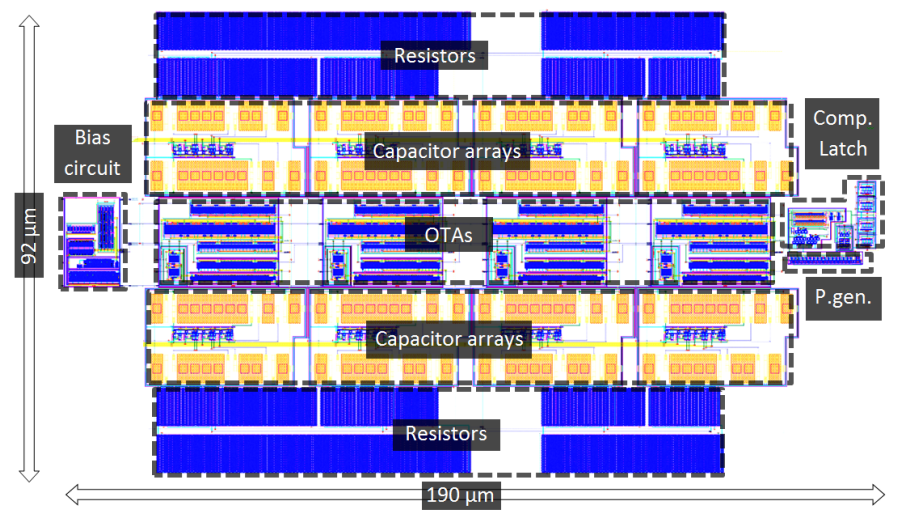

Fig. 9 Layout of the CTDS ADC designed.

\section{CTDS ADC simulations}

After the assembly of all the blocks, the layout of the full CTDS ADC, with a total area of $0.0175 \mathrm{~mm}^{2}$ is shown in Fig. 9. The area distribution is as follows: OTAs, including its bias circuit, occupy $3100 \mu \mathrm{m}^{2}(17.7 \%)$, the capacitor arrays $7600 \mathrm{\mu m}^{2}(43.4 \%)$, the coefficient resistors $6300 \mathrm{\mu m}^{2}(36 \%)$, and the pulse generator, comparator, latch and DACs combined occupy $500 \mu \mathrm{m}^{2}$ $(2.9 \%)$. It can be seen that the majority of the area is occupied by the loop filter, and the area of the quantizer is significantly smaller. In order to achieve better matching, the resistors and capacitors were split in several unit size devices and interleaved. Moreover, dummy resistors and capacitors were placed around each matching structure. Similarly, the transistors were matched using common-centroid techniques and dummy devices.

The output spectrum of the full CTDS ADC with extracted parasitics (PEX) is shown in Fig. 10. Note that due to the optimal zero placing and the even order of the CTDS ADC, there are no zeros at DC. The nominal SNR and current consumption simulated with extracted parasitics are $45 \mathrm{~dB}$ and $489 \mu \mathrm{A}$ respectively. From the total current, $443 \mu \mathrm{A}$ are spent on the OTAs $(90.6 \%), 22 \mu \mathrm{A}$ are spent on the quantizer (4.5\%) and $24 \mu \mathrm{A}$ are spent in the DACs $(4.9 \%)$. The current consumption is clearly dominated by the loop filter, mainly in the OTAs. The supply voltage is $1.2 \mathrm{~V}$, hence the power consumption of the CTDS ADC results in $0.587 \mathrm{~mW}$.

The CTDS ADC is also simulated with extracted parasitics in the corners and with temperature and supply variations. A summary of the typical, maximum and minimum values obtained over all combinations are shown in Table 3. The capacitor array is adjusted for each simulation to compensate for the capacitance variation and adjust the loop filter coefficients accordingly. The design falls within specifications even across the corners and variations. 


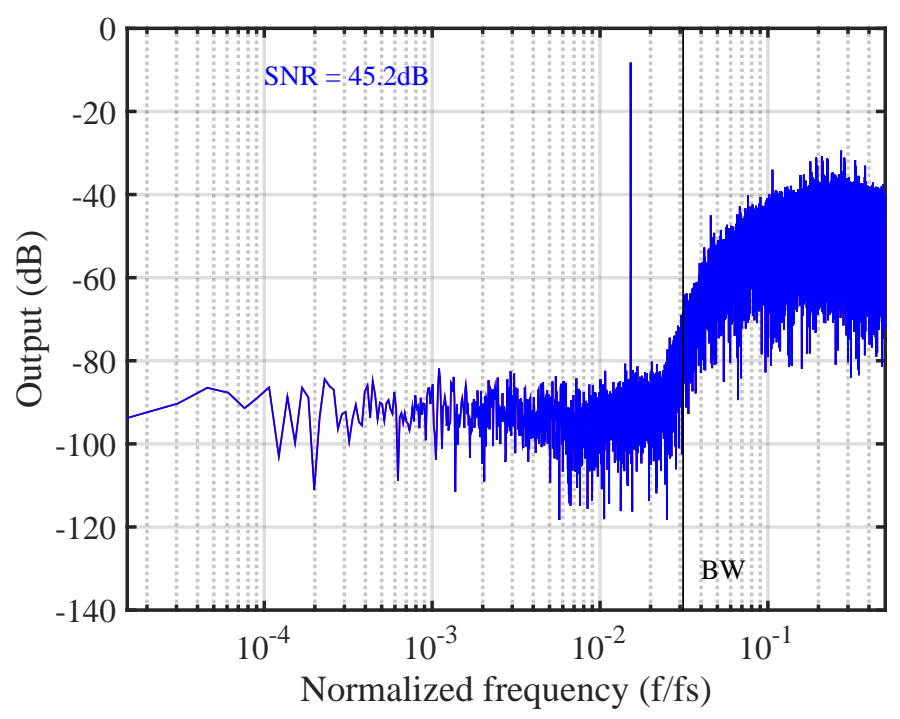

Fig. 10 Simulated frequency response of the CTDS ADC with an input amplitude of $u_{i n}$ $=0.6 \mathrm{~V}$ with parasitic extraction (PEX). Nominal corner.

Table 3 CTDS ADC Performance over Corners and Variations

\begin{tabular}{lll}
\hline & SNR $[\mathrm{dB}]$ & $\mathrm{P}[\mathrm{mW}]$ \\
\hline Nom. & 45.2 & 0.587 \\
Min. & 42.7 & 0.540 \\
Max. & 47.1 & 0.639 \\
\hline
\end{tabular}

\section{Results and discussion}

The CTDS ADC was fabricated in a $65 \mathrm{~nm}$ process, and a die picture of the integrated circuit (IC) taken with a microscope is shown in Fig. 11. A printed circuit board (PCB) was designed to test the circuit. The PCB contains lowdrop out (LDO) voltage regulators and decoupling capacitors to stabilize the circuit references and supply voltages. The PCB voltages are fed using two Rigol DP832 programmable DC power supplies. The master clock of the CTDS ADC is supplied with a low jitter, high-accuracy clock generator AD9516-3. The differential input signals are generated with a Tektronix AFG3102C function generator and the differential outputs of the ADC are measured using Rohde \& Schwarz RTO 1024 oscilloscope. A picture of the measurement setup can be seen in Fig. 12. For the purpose of comparing the simulations with the measurement results accurately, the clock jitter, supply variations and the parasitic resistances, capacitances and inductances introduced by the measurement setup were estimated and modeled. The most relevant parasitics in the setup are the coupling capacitances from the ESD protection of the pads, the inductances and resistances from the bondwires and IC socket and the 


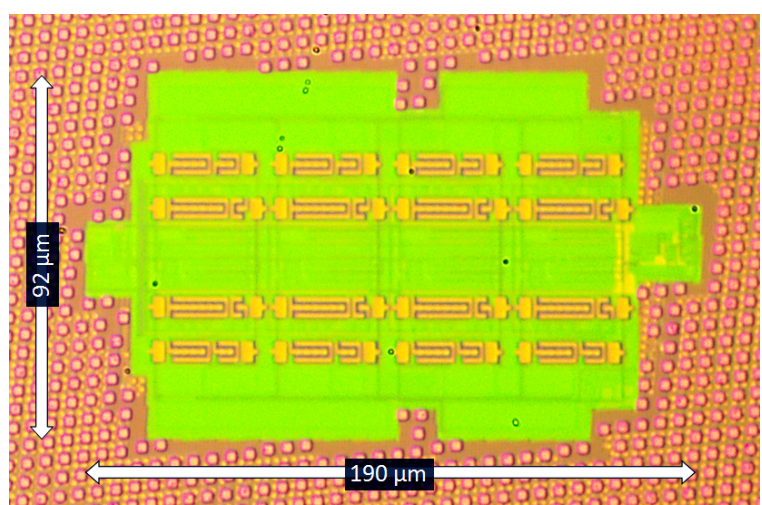

Fig. 11 Picture of the fabricated die containing the continuous-time delta-sigma ADC.

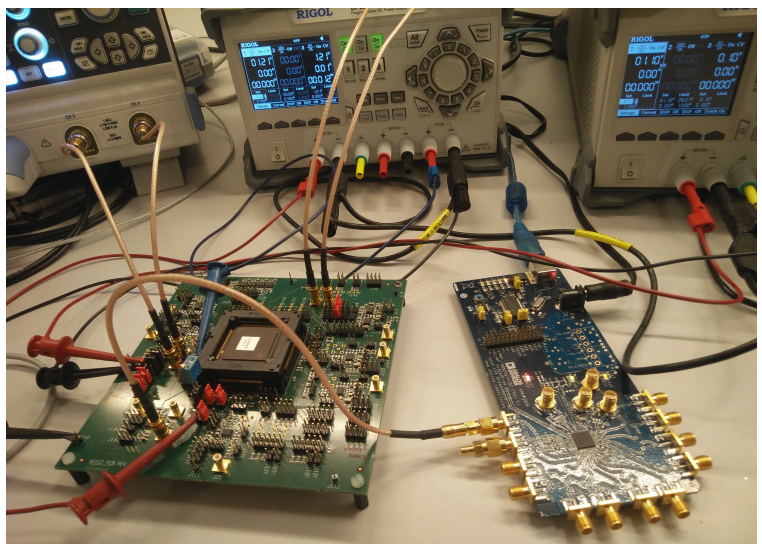

Fig. 12 Continuous-time delta-sigma ADC measurement setup.

resistance of the traces in the $\mathrm{PCB}$. The model of the measurement setup was added to the simulations obtaining a SNR of $42.1 \mathrm{~dB}$ and a current consumption of $491 \mu \mathrm{A}$, which is the expected SNR to be measured in the IC. The SNR degradation due to the measurement setup and packaging is estimated to be $3.1 \mathrm{~dB}$. The simulations show that this degradation is mainly caused by the inductances from the package and IC socket, which create voltage bouncing on the references $V_{R E F+} / V_{R E F-}$. This phenomenon will not be an issue on the final IC since the supplies will be on-chip. Hence, this degradation is only present due to the fact that the $\mathrm{ADC}$ is tested as an isolated block and not part of the system. However, if this degradation had to be overcome in this current IC, the die would need to be flip-chip bonded into a PCB directly to avoid packaging.

The frequency response measured on the IC (Meas.) can be seen in Fig. 13. Additionally, the simulations with extracted parasitics (PEX), and the simulations with extracted parasitics and measurement setup modeled ( $\mathrm{PEX}^{*}$ ) are shown to ease the comparison. The frequency response and SNR fit closely 


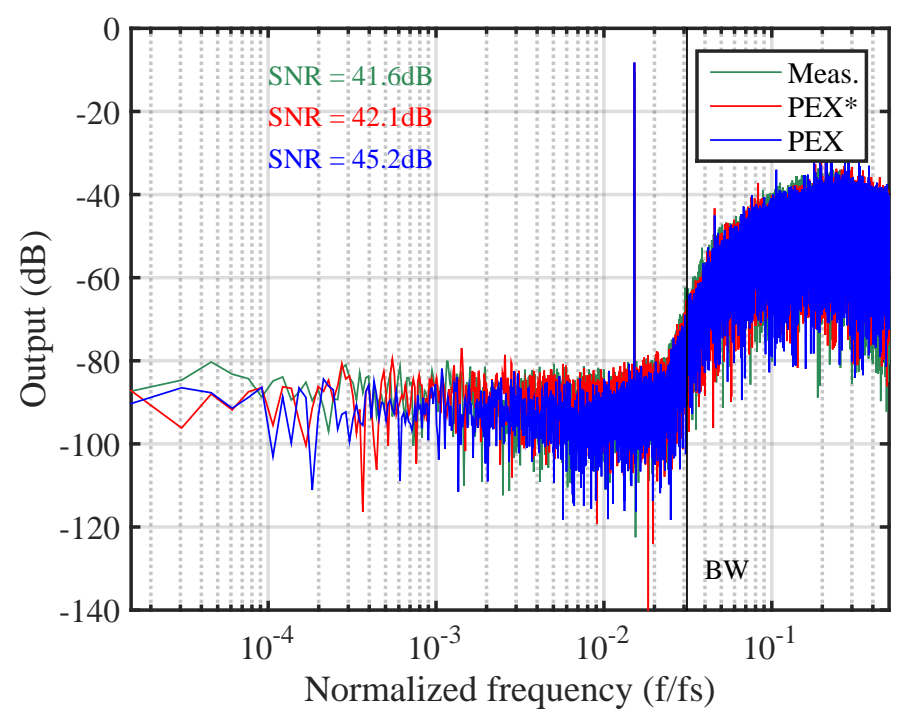

Fig. 13 Frequency response of the CTDS ADC with an input amplitude of $u_{i n}=0.6 \mathrm{~V}$. Measurements (Meas.), simulated results with parasitic extraction and measurement setup modeled $\left(\mathrm{PEX}^{*}\right)$ and simulated results with parasitic extraction $(\mathrm{PEX})$.

with the simulated results with the measurement setup modeled. Furthermore, the measured current consumption is $495 \mu \mathrm{A}$, which is also very close to the simulated $491 \mu \mathrm{A}$. The CTDS ADC is designed to be connected inside a die, without receiving or delivering any outputs directly outside of the IC. Consequently, when the circuit is used in a portable ultrasound scanner, the SNR degrading effects caused by the measurement setup would not be present. Due to the high correlation from simulations and measurements, the CTDS ADC is expected to operate inside an Rx channel with a performance similar to the simulations with extracted parasitics, $45 \mathrm{~dB}$.

In order to assess the SNR and power consumption $\left(P_{A D C}\right)$ variation spread over several dies, the approach suggested in [12] is used. In Fig. 14, the measured SNR and power consumption of 20 dies and their respective median range, $M$, and percentiles $P_{15.87}$ and $P_{84.13}$ for a confidence level of $95 \%$ are shown. The SNR performance spread is proportionally small with $P_{15.87}$ and $P_{84.13}$ located at $41.05 \mathrm{~dB}$ and $42.51 \mathrm{~dB}$ respectively, hence, a consistent performance across dies is expected. The power consumption spread is also low with $P_{15.87}$ and $P_{84.13}$ located at $0.548 \mathrm{~mW}$ and $0.636 \mathrm{~mW}$ respectively, but more significant than the SNR spread. This is attributed to the OTAs biasing circuit, where small transistors were used to reduce the parasitic capacitances and thereby the power consumption. As a result, the die variations affect the total power consumption of the CTDS ADC more significantly. In the future, a biasing circuit with higher variation tolerance will be investigated.

A performance comparison between this design and other CTDS ADC with similar specifications is provided in Table 4, where the standard figure 

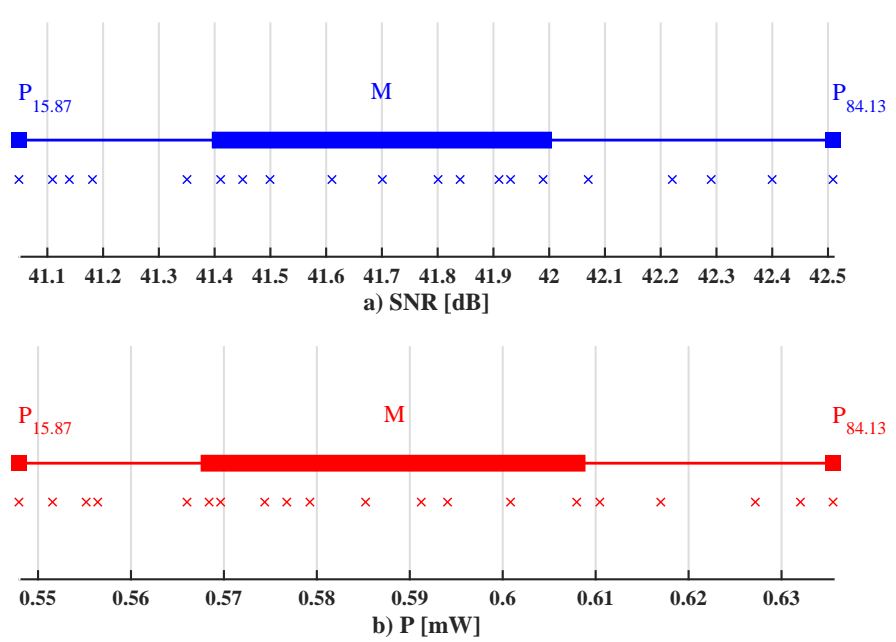

Fig. 14 Data sets of SNR and P for a 95\% confidence level, showing the spread of the median $M$ and the percentiles $P_{15.87}$ and $P_{84.13}$ for $N=20$.

of merit (FoM) of energy per conversion is used (1). Both the measured performance (Meas.), and the expected performance without the measurement setup SNR degradation (PEX) are included. As it can be seen, this design achieves a comparatively low FoM using a very small die area and low power consumption which enables channel scalability, a necessary factor for portable ultrasound scanners. Note that the die area, which is one of the strengths of this design, is not part of the FoM, hence it is not taken into account for the comparison.

$$
F o M=\frac{P_{A D C}}{2 \cdot B W \cdot 2^{\frac{S N R-1.76 d B}{6.02 d B}}}
$$

In order to put in perspective the power consumption of the CTDS ADC in the portable ultrasound scanner, the full system is considered. A 64-channel portable ultrasound scanner, containing $64 \mathrm{ADCs}$, has an approximate total power budget of $2 \mathrm{~W}$. Using 64 of the designed CTDS ADC, only a power consumption of $38 \mathrm{~mW}$ is used which is a very small portion of the total budget. As a result, this efficient ADC implementation allows for most of the power budget to be spent in the other parts of the scanner.

\section{Conclusions}

In this paper a fully differential fourth-order 1-bit continuous-time delta-sigma ADC designed in a $65 \mathrm{~nm}$ process for portable ultrasound scanners is presented. The ADC has a supply voltage of $1.2 \mathrm{~V}$, a BW of $10 \mathrm{MHz}$, and operates at a $f_{s}$ of $320 \mathrm{MHz}$. The aim of the design is to reduce the area and minimize the power consumption achieving a small, efficient and scalable design that 
Table 4 CTDS ADC comparison

\begin{tabular}{cllllll}
\hline & This work & {$[13]$} & {$[14]$} & {$[15]$} & {$[16]$} & {$[17]$} \\
\hline SNR [dB] & $45.2 / 41.6^{*}$ & 54.5 & 44 & 64.5 & 67.9 & 70 \\
BW [MHz] & 10 & 5 & 20 & 20 & 10 & 10 \\
Fs [MHz] & 320 & 200 & 522 & 640 & 320 & 300 \\
Area [mm $\left.{ }^{2}\right]$ & 0.0175 & - & - & 0.072 & 0.39 & 0.051 \\
Power [mW] & $0.587 / 0.594^{*}$ & 3.4 & 11.6 & 11 & 4.8 & 2.57 \\
FoM [fJ/c.] & $197 / 302^{*}$ & 360 & 1900 & 225 & 230 & 50 \\
\hline * & & & & &
\end{tabular}

*Measured degraded performance.

can be used in a portable ultrasound scanner. Due to the low SNR specifications, the design is inherently dominated by quantization noise, which is uncommon for CTDS ADC and expands the possible design space. The loop filter uses OTA based RC-integrators and voltage feedback DACs. The quantizer is composed of a high-speed clocked comparator and a pull-down clocked latch which are both controlled by a pulse generator. The design occupies a die area of $0.0175 \mathrm{~mm}^{2}$ and is robust to corners and process variations. The power consumption measured on the fabricated IC is $0.594 \mathrm{~mW}$, and the measured SNR degraded by the measurement setup is $41.6 \mathrm{~dB}$, leading to a FoM of $302 \mathrm{fJ} /$ conversion. However, the expected SNR of the CTDS ADC used internally on an IC, without measurement setup degradation, is $45 \mathrm{~dB}$, leading to a FoM of $197 \mathrm{fJ} /$ conversion. Measurements across several dies show a consistent design with a small SNR die spread.

\section{References}

1. Richard Schreier and Gabor C. Temes. Understanding Delta-Sigma Data Converters. IEEE, 2004.

2. M. Ortmanns and F. Gerfers. Continuous-Time Sigma-Delta A/D Conversion. Springer, 2005.

3. Tommaso Di Ianni, Martin Hemmsen, Pere Llimós Muntal, Ivan H. H. Jørgensen, and Jørgen Jensen. System-level Design of an Integrated Receiver Front-end for a Wireless Ultrasound Probe. IEEE Transactions on Ultrasonics, Ferroelectrics, and Frequency Control, 63(11):1935-1946, 2016.

4. Jacob Kortbek, Jorgen Arendt Jensen, and Kim Lokke Gammelmark. Synthetic Aperture Sequential Beamforming. In 2008 IEEE Ultrasonics Symposium, number 1, pages 966-969. IEEE, 2008.

5. Martin Christian Hemmsen, Peter Møller Hansen, Theis Lange, Jens Munk Hansen, Kristoffer Lindskov Hansen, Michael Bachmann Nielsen, and Jørgen Arendt Jensen. In Vivo Evaluation of Synthetic Aperture Sequential Beamforming. Ultrasound in Medicine \& Biology, 38(4):708-716, 2012.

6. Martin Christian Hemmsen, Jens Munk Hansen, and Jørgen Arendt Jensen. Synthetic aperture sequential beamformation applied to medical imaging. In EUSAR 2012, 2012.

7. Jacob Kortbek, Jørgen Arendt Jensen, and Kim Løkke Gammelmark. Sequential beamforming for synthetic aperture imaging. Ultrasonics, 53(1):1-16, 2013.

8. Pere Llimós Muntal, Kjartan Færch, Ivan H. H. Jørgensen, and Erik Bruun. System level design of a continuous-time $\Delta \Sigma$ modulator for portable ultrasound scanners. In 2015 Nordic Circuits and Systems Conference (NORCAS): NORCHIP \& International Symposium on System-on-Chip (SoC), pages 1-4. IEEE, 2015. 
9. Niels Marker-Villumsen and Erik Bruun. Optimization of modulator and circuits for low power continuous-time Delta-Sigma ADC. NORCHIP 2014 - 32nd NORCHIP Conference: The Nordic Microelectronics Event, 2015.

10. Willy M. C. Sansen. Analog Design Essentials, volume 859. Springer US, Boston, MA, 2006

11. U. K. Vijay and Amrutur Bharadwaj. Continuous time sigma delta modulator employing a novel comparator architecture. Proceedings of the IEEE International Conference on VLSI Design, (Figure 1):919-924, 2007.

12. Hanspeter Schmid and Alex Huber. Measuring a small number of samples, and the 3v fallacy: Shedding light on confidence and error intervals. IEEE Solid-State Circuits Magazine, 6(2):52-58, 2014.

13. Pengyu Song, Kei Tee Tiew, Yvonne Lam, and Liang Mong Koh. A CMOS 3.4 mW 200 $\mathrm{MHz}$ continuous-time delta-sigma modulator with $61.5 \mathrm{~dB}$ dynamic range and $5 \mathrm{MHz}$ bandwidth for ultrasound application. Midwest Symposium on Circuits and Systems, pages 152-155, 2007.

14. Young Kyun Cho, Sung Jun Lee, Seung Hyun Jang, Bong Hyuk Park, Jae Ho Jung, and Kwang Chun Lee. 20-MHz bandwidth continuous-time delta-sigma modulator for EPWM transmitter. Proceedings of the International Symposium on Wireless Communication Systems, pages 885-889, 2012.

15. Xiaodong Liu, Mattias Andersson, Martin Anderson, Lars Sundstrom, and Pietro Andreani. An $11 \mathrm{~mW}$ continuous time delta-Sigma modulator with $20 \mathrm{MHz}$ bandwidth in 65nm CMOS. In 2014 IEEE International Symposium on Circuits and Systems (ISCAS), number 2, pages 2337-2340. IEEE, 2014

16. Yang $\mathrm{Xu}$, Zehong Zhang, Baoyong Chi, Qiongbing Liu, Xinwang Zhang, and Zhihua Wang. Dual-mode $10 \mathrm{MHz}$ BW $4.8 / 6.3 \mathrm{~mW}$ reconfigurable lowpass/complex bandpass CT sigma-delta modulator with 65.8/74.2dB DR for a zero/low-IF SDR receiver. In 2014 IEEE Radio Frequency Integrated Circuits Symposium, pages 313-316. IEEE, 2014.

17. Kazuo Matsukawa, Koji Obata, Yosuke Mitani, and Shiro Dosho. A 10 MHz BW 50 fJ/conv. continuous time sigma-delta; modulator with high-order single opamp integrator using optimization-based design method. In 2012 Symposium on VLSI Circuits (VLSIC), pages 160-161. IEEE, 2012. 